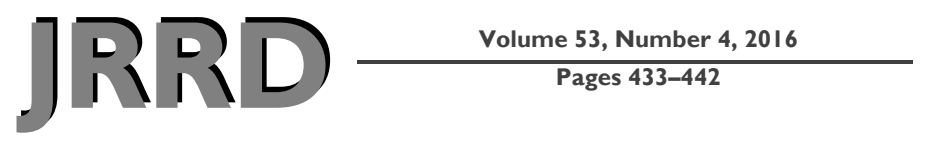

\title{
Interdisciplinary development of an ergonomic prone mobility cart
}

\author{
Steven W. Brose, DO; ${ }^{1-2^{*}}$ Martin J. Kilbane, PT, DPT, OCS; ${ }^{1}$ Elizabeth Harpster, BA; ${ }^{2}$ Steven J. Mitchell, \\ OTR/L, ATP; ${ }^{1}$ Chester Ho, MD; ${ }^{3}$ Ken J. Gustafson, PhD $^{1}$ \\ ${ }^{1}$ Spinal Cord Injury Unit, Louis Stokes Cleveland Department of Veterans Affairs Medical Center, Cleveland, OH; \\ ${ }^{2}$ Department of Physical Medicine and Rehabilitation, Case Western Reserve University School of Medicine, Cleve- \\ land, $\mathrm{OH} ;{ }^{3}$ Department of Clinical Neurosciences, Division of Physical Medicine and Rehabilitation, University of \\ Calgary, Calgary, Canada
}

\begin{abstract}
Pressure ulcers remain a major source of morbidity and mortality in Veterans with neurologic impairment. Management of pressure ulcers typically involves pressure relief over skin regions containing wounds, but this can lead to loss of mobility and independence when the wounds are located in regions that receive pressure during sitting. An innovative, iterative design process was undertaken to improve prone cart design for persons with spinal cord injury and pressure ulceration. Further investigation of ways to improve prone carts is warranted to enhance the quality of life of persons with pressure ulcers.
\end{abstract}

Key words: cart, decubitus, design, iterative, pressure, pressure ulcer, prone, spinal, spinal cord injury, ulcer.

\section{INTRODUCTION}

Pressure ulcers frequently occur in the setting of spinal cord injury (SCI) [1-3] and can decrease quality of life, lengthen hospitalization, increase care requirements, and lead to subsequent medical complications [4-7]. A key component of managing pressure ulcers is reducing pressure in the region of the wound [8], which can involve decreased or eliminated sitting time in ischial and sacral ulcers; however, prolonged bedrest can lead to deconditioning, respiratory compromise, and significant psychosocial impact [5]. Use of traditional manual prone carts can relieve pressure on the ischium and sacrum while improving mobility and social interaction, but it has been observed that these carts frequently cause neck, shoulder, and back pain [9]. These problems have led to several attempts to develop solutions, including more ergonomically tilted carts [9] and various designs of motorized carts [10-11], as well as a recent report we published in the Journal of Rehabilitation Research and Development describing an effort to convert a power chair into a motorized prone cart that faced a variety of challenges [12]. This article describes the iterative, interdisciplinary process of developing an ergonomic, motorized, prone mobility cart over a period of several years and multiple design iterations for quality improvement of clinical care for pressure ulcers.

Abbreviations: SCI = spinal cord injury, VA = Department of Veterans Affairs, VAMC = VA medical center.

*Address all to correspondence Steven W. Brose, DO; Louis Stokes Cleveland VAMC, 10701 East Blvd, Cleveland, OH 44106; 216-791-3800, ext 3658; fax: 216-7075936. Email: steven.brose@va.gov

http://dx.doi.org/10.1682/JRRD.2014.11.0279 


\section{ITERATIVE DESIGN PROCESS AND CLINICAL OUTCOMES}

The process of developing a commercial-grade, motorized, prone mobility cart began with the basic prone cart design commonly used in hospitals nationwide. Prone carts were heavily used at the Department of Veterans Affairs (VA) SCI Service in which this work took place because of the high pressure ulcer prevalence in this population. During the evolution of the prone cart design, interdisciplinary teams focused on a series of key goals: motorization, maneuverability, ergonomic fit, and utility to support functional independence (Figure 1). The first design iterations involved addressing the shoulder pain associated with use of the standard manual prone mobility cart by adding power function and increasing maneuverability. After these modifications, users continued to report discomfort in and around their cervical and thoracic spines, citing strain associated with lifting their heads up to see while driving the cart. Collaboration with engineers at a major academic center allowed development of a more ergonomic support surface and incorpora- tion of utility items for functional independence. Further design in collaboration with industry representatives allowed for creation of a commercial-grade ergonomic motorized prone cart (Table).

Each prone cart iteration was used by between four and six different persons with SCI who were at that time located on the inpatient SCI ward at Louis Stokes Cleveland VA Medical Center (VAMC). During this design process, persons with SCI were continually evaluated by physical therapy using the Louis Stokes Cleveland VAMC Spinal Cord Injury/Disorder Prone Cart Protocol (Figure 2). The protocols for trialing the designs were put in place to determine whether persons with SCI were able to tolerate the prone position before use of the cart and to allow users ample time to get used to the functions of each prone cart. Users were first evaluated by physical therapy for their ability to tolerate the prone position while lying in bed for increasing time increments over a 4-day period. Once it was determined that these users were able tolerate the prone position in bed, they were transitioned to use of the prone cart, again over a period of 2 days, with increasing time and close supervision by a

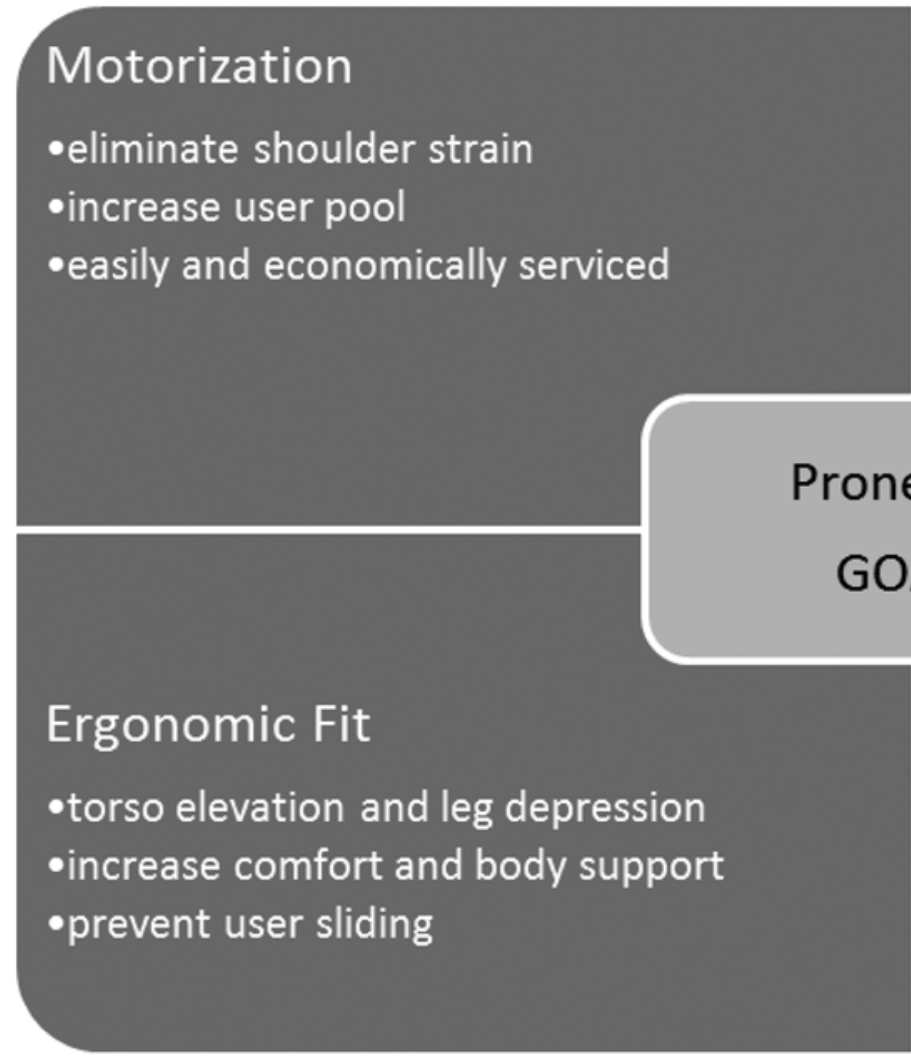

\section{Maneuverable}

-decrease turning radius

-reduce footprint

•lightweight

Prone Cart

GOALS

Utility

- addition of convenience items (cupholder,tray)

- cutouts for catheter

•adjustable height

\section{Figure 1.}

Interdisciplinary goals of prone cart design. 
Table.

Comparison of prone cart iterations.

\begin{tabular}{|c|c|c|c|c|c|}
\hline & Iteration 1 & Iteration 2 & Iteration & Iteration 4 & $\begin{array}{c}\text { Future } \\
\text { Iteration (5) }\end{array}$ \\
\hline Time & Pre-2007 & 2007-2012 & 2010 & 2013-present & Future suggestions \\
\hline Motion & Manual & E-fix motorized & Manual & E-fix motorized & Motorized \\
\hline Frame & Rectangular & H-frame & Rectangular & H-frame & $\begin{array}{l}\text { H-frame with rounded } \\
\text { edges }\end{array}$ \\
\hline Cutouts & None & None & Foot & $\begin{array}{l}\text { Arms, chin, foot, catheter/ } \\
\text { abdominal }\end{array}$ & $\begin{array}{l}\text { Increased cutout sizes } \\
\text { for arms, chin, foot, } \\
\text { catheter/abdominal }\end{array}$ \\
\hline
\end{tabular}

therapist. Finally, users were able to maneuver the prone cart independently on the ward for increasing time increments of 30 to $60 \mathrm{~min}$ and eventually able to travel throughout the hospital (Figure 3). Each user's total time with different prone cart iterations varied throughout the design process as each user's ability to tolerate the prone position and maneuver the cart was unique. As this was a quality improvement project, the feedback received from persons with SCI on the use of the prone carts was conducted verbally though interviews with users and their therapists, nurses, and physicians. User feedback was crucial in the redesign of each prone cart iteration.

\section{Iteration 1: Standard Prone Cart}

\section{Cart Specifications and Design Goals}

- Height: $61 \mathrm{~cm}$ tall.

- Wheels: $56 \mathrm{~cm}$ spoke wheels.

- Mobility: Manual.

- Frame: 115 kg, rectangular.

- Design goal: Original cart.

\section{Design}

The original prone cart used on the VA SCI service prior to 2007 (when this work began) was a flat bed with manual wheelchair wheels and straps to secure the user (Figure 4). The cart lacked a tray and was thus incapable of storing and holding items for persons with SCI using the cart. The height was relatively short at only $61 \mathrm{~cm}$.

\section{User Response}

In order to make use of this unmodified cart, persons with SCI and pressure ulceration needed to be able to tolerate the prone position, be able to support themselves on their elbows, and be able to hold their head up while pushing the cart manually. This version had little consideration for ergonomic design, which created many user complaints of shoulder, neck, and back pain, in accordance with established literature [9]. These many complaints, stemming from frequent use of the cart, stimulated the modifications described in this article. The original cart required users to push at a shoulder angle of more than $90^{\circ}$, causing increased strain on the shoulder joint. It was very difficult to maneuver through hallways 

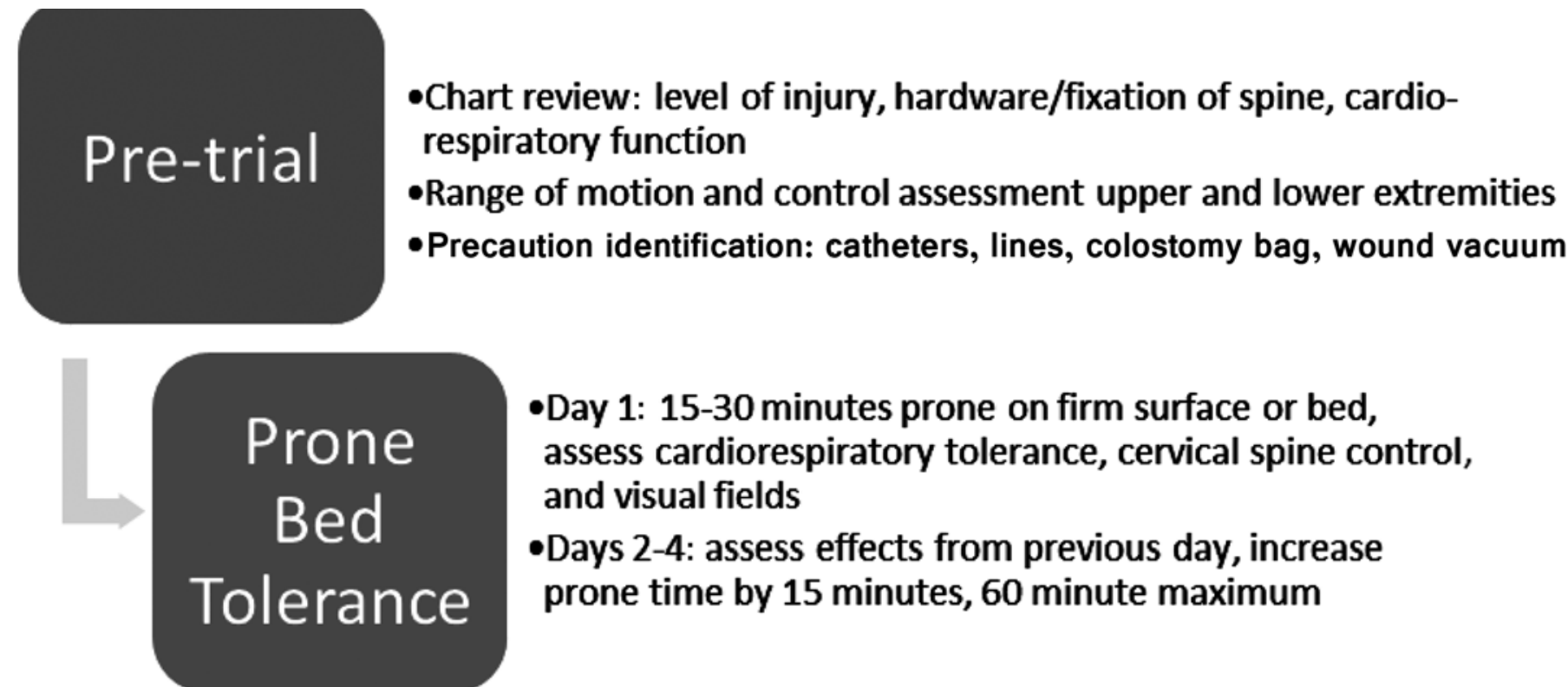

-Day 1: 15-30 minutes prone on firm surface or bed, assess cardiorespiratory tolerance, cervical spine control, and visual fields

-Days 2-4: assess effects from previous day, increase prone time by 15 minutes, 60 minute maximum

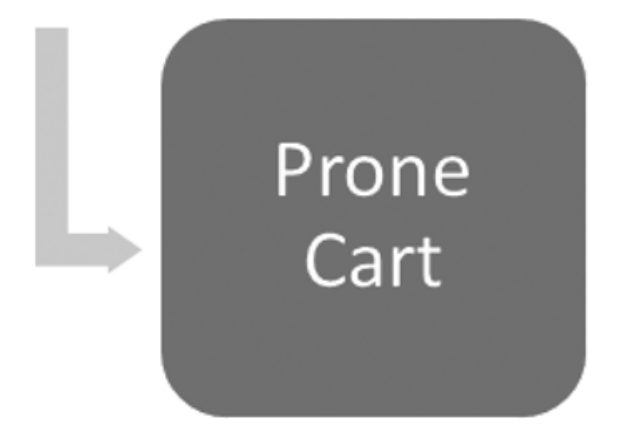

-Day 5-6: prone cart training with therapist

-Day 7+: independent use by patient, add 30-60 minutes every 3 days

Figure 2.

Prone cart evaluation protocol.

and elevators because the turning radius was almost the length of the entire cart.

The cart lacked a tray to hold items such as drinks, plates, books, or computers, thus limiting potential benefits associated with using the cart. The short height of the cart (only $61 \mathrm{~cm}$ ) made it harder for users to see their environment because of being close to the ground. This height also made bed-level transfers difficult because the cart lacked the ability to adjust in height to match hospital bed height.

\section{Iteration 2: Motorization and Base Development}

Cart Specifications and Design Goals

\section{First Motorized}

- Height: $61 \mathrm{~cm}$ tall.

- Wheels: $61 \mathrm{~cm}$ rear spoke wheels.
- Mobility: E-fix motorized system.

- Frame: 115 kg, rectangular.

- Design goal: Add motorization.

\section{Second Motorized}

- Height: $61 \mathrm{~cm}$ tall, with $15 \mathrm{~cm}$ of additional adjustable height.

- Wheels: $30.5 \mathrm{~cm}$ rubber wheels, recessed.

- Mobility: E-fix motorized system.

- Frame: 70 kg, H-frame.

- Design goal: Increase maneuverability and utility for functional independence.

Design

From 2007 to 2012, an interdisciplinary team of engineers, therapists, physicians, and industry manufactures worked to improve motorization and maneuverability of 


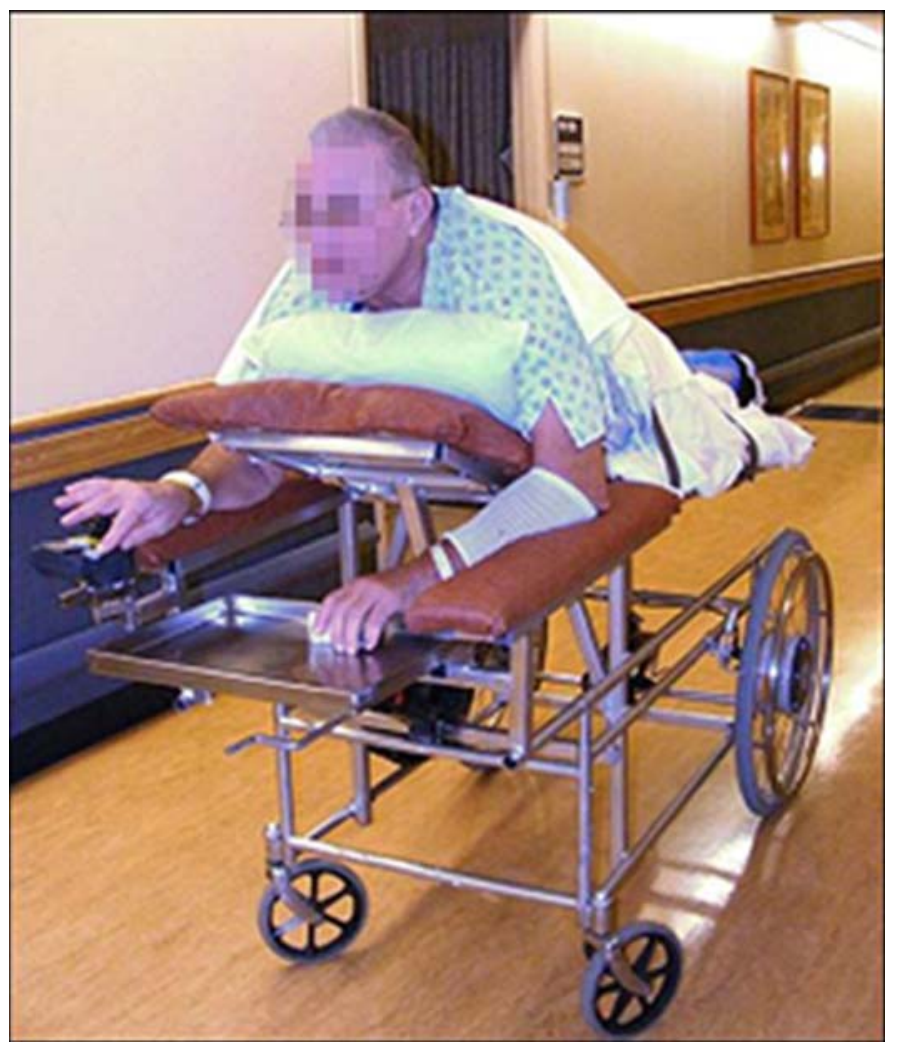

Figure 3.

Person with spinal cord injury using a prone cart.

the device, applying feedback from users. This design team first attempted to better manage the shoulder strain encountered by users of the prone cart through the addition of a motorized system. The first attempt was the addition of power assist to the $61 \mathrm{~cm}$ spoke wheels. However, the cart was difficult to maneuver in a straight line and tended to have a fish-tailing motion. Next, an E-fix system was placed on the original cart (used for several years prior to 2007) (Figure 5). While this design alleviated shoulder strain from pushing the cart, it did not achieve the goals of maneuverability and ergonomic design. The spoke wheels located in the rear of the cart were easy targets for damage against rails and corners. One user of this cart damaged the spokes while attempting to maneuver through elevators at the VA; these wheels, once damaged, were difficult to service and replace.

The final and more maneuverable wheel base incorporated a new $\mathrm{H}$-frame and $30.5 \mathrm{~cm}$ tire wheels, which were also used in motorized wheelchair units (Figure 6).

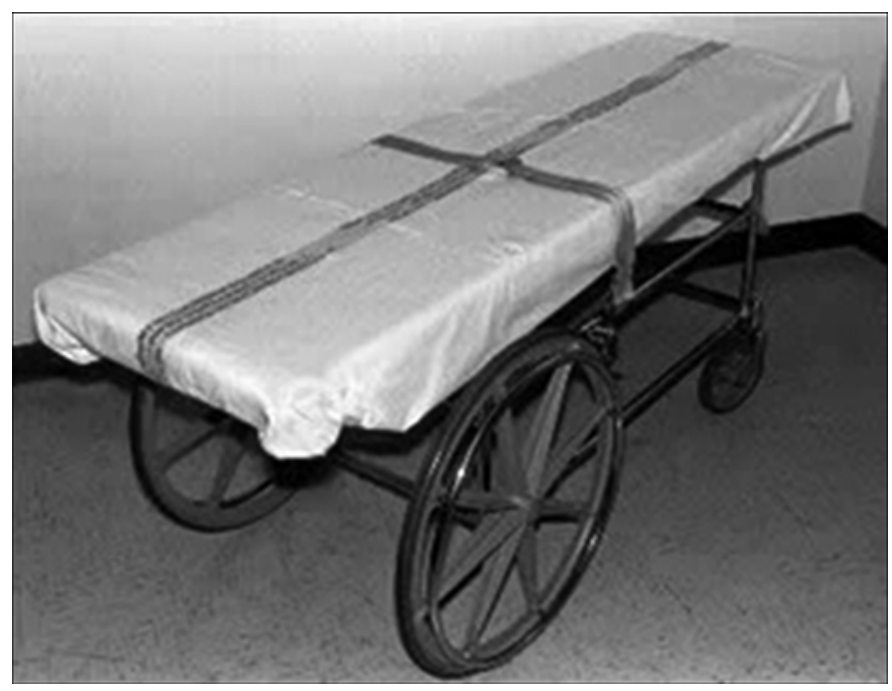

Figure 4.

Iteration 1: Standard prone cart.

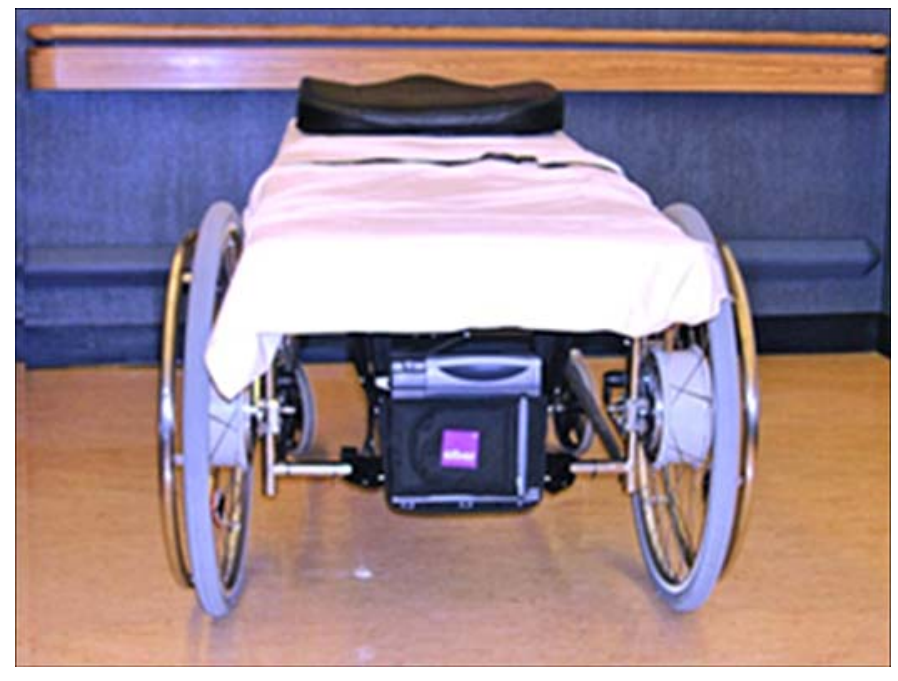

Figure 5.

Iteration 2: E-fix system.

The new frame and smaller wheels significantly reduced the weight of the prone cart from approximately $115 \mathrm{~kg}$ down to about $70 \mathrm{~kg}$. This new frame and wheel base allowed for recessed wheels under the support surface of the cart, reducing the prone cart footprint for transfers and turning radius while increasing the durability of the structure (Figure 7). The motor and wheels for the prone cart were used in other motorized wheelchairs, so these parts were easily serviced by VA technicians. Tilt supports 


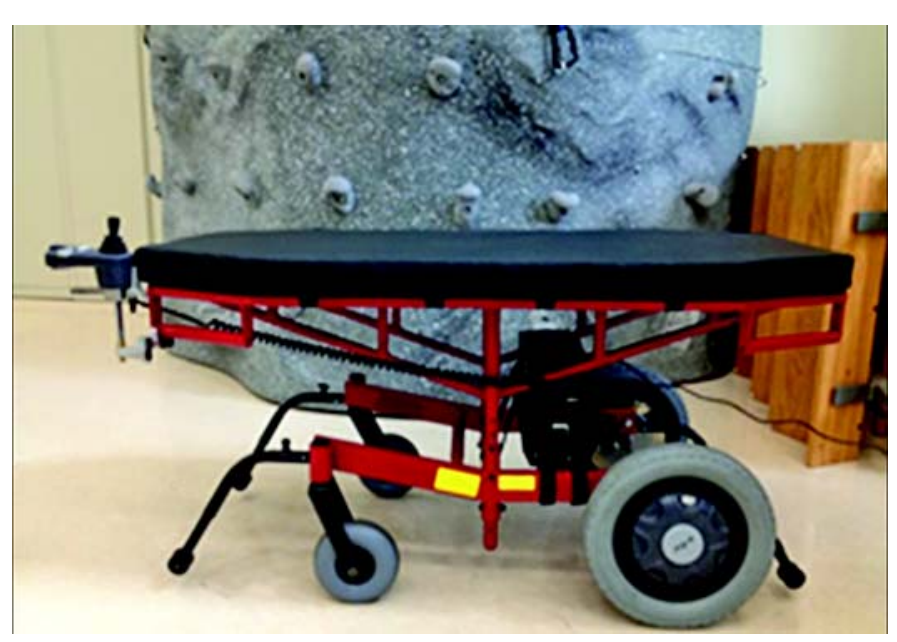

Figure 6.

Iteration 2: Incorporation of $\mathrm{H}$-frame and $30.5 \mathrm{~cm}$ tire wheels.

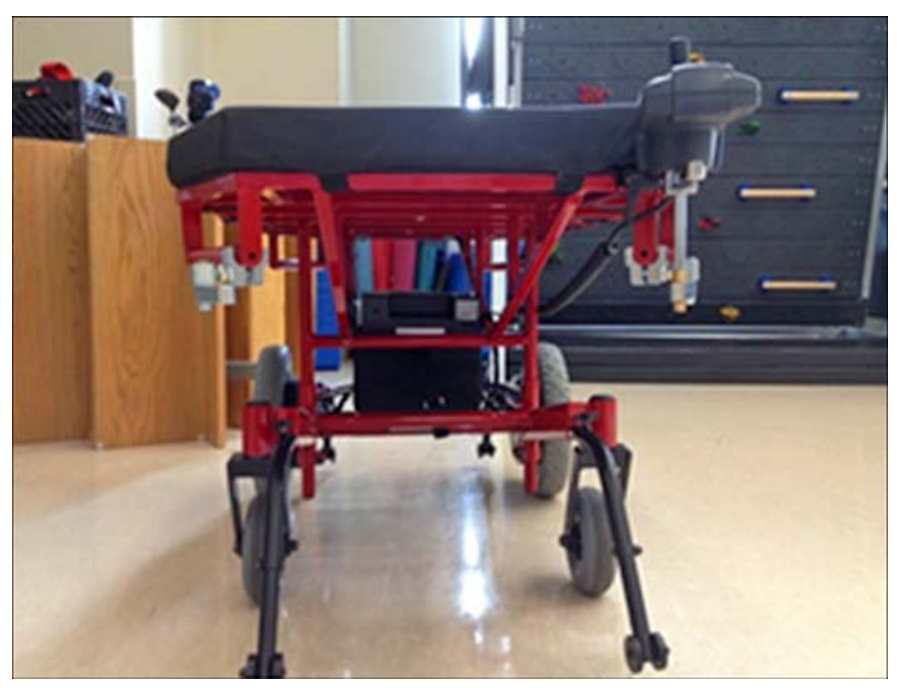

Figure 7.

Alternate view of incorporation of $\mathrm{H}$-frame and $30.5 \mathrm{~cm}$ tire wheels.

were added to this design as a safety precaution against tipping. The new $\mathrm{H}$-frame also added an adjustable feature that allowed the prone cart about $15 \mathrm{~cm}$ of adjustable height to customize the prone cart for bed-level transfers.

\section{User Response}

The new motorized component, smaller wheel base, and better turning radius made this updated design more maneuverable and useful for persons with SCI. Therapists noted that users were able to travel through hall- ways, around corners, and through the elevators easier and without damage to the cart. The bed-level transfers were safer and easier with the adjustable height feature that allowed for a better match with hospital bed heights at the VA. The E-fix motorized component prevented shoulder strain on the user by removing the pushing requirement. Because the new motorized system and wheels were also used on other motorized wheelchairs in use at the VA, the new prone cart was economical to service.

While this cart met goals of motorization and increased maneuverability, it still did not address the ergonomics of the support surface. Users still had difficulty looking forward and needed to be able to support themselves on their elbows or elevate their head while driving the cart. This cart also lacked convenience features that users felt would better serve them while on the cart, such as a tray and cup holder.

\section{Iteration 3: Ergonomic Support Surface}

\section{Cart Specifications and Design Goals}

- Height: $76 \mathrm{~cm}$ tall.

- Wheels: $61 \mathrm{~cm}$ spoke wheels.

- Mobility: Manual.

- Frame: Rectangular with split cart pad able to change angles.

- Design goal: Better ergonomic fit and increased utility.

\section{Design}

Collaboration in 2010 between engineers at a major academic institution and SCI therapists led to the design of a support surface that was a better ergonomic fit for users. Previous designs such as the Sammy LS Prone Cart (Figure 8) attempted to angle the users upward with a single support pad to allow easier visibility; however, users reported that the straight support pad caused a sensation of sliding down the cart when the pad was elevated. Applying experience from previous prone carts and user feedback, the team created a split design in which the user could both elevate the torso pad and decline the leg pad (Figure 9). The new ergonomic support surface helped prevent undue strain to the neck and back. The torso pad was able to be elevated to $40^{\circ}$, and the leg pad was able to decline to $-30^{\circ}$. The design team added two safety belts to secure the user on the cart as well as a foot cutout and foot strap to prevent sliding down the cart. Convenience and utility items were added that included arm rests, a cup holder, and a tray. 


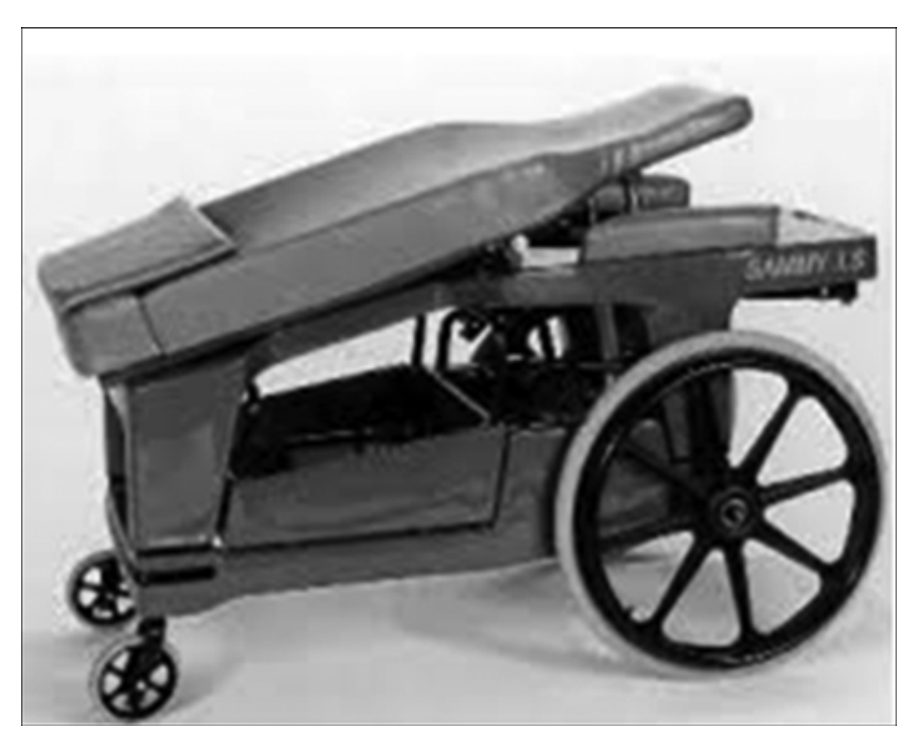

Figure 8.

Sammy LS prone cart.

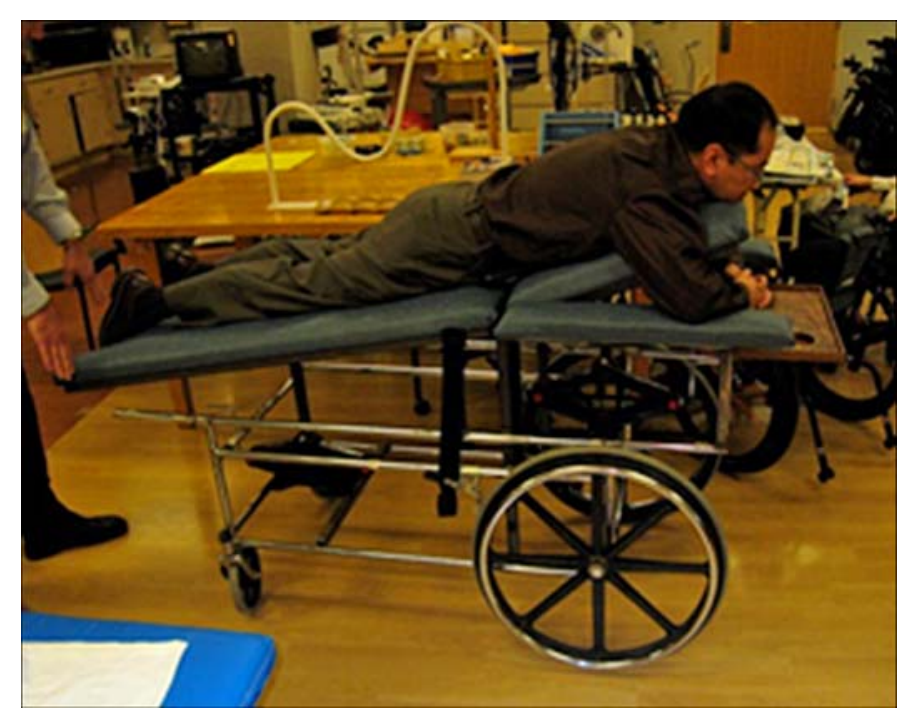

Figure 9.

Iteration 3: Ergonomic support surface, highlighting split design.

\section{User Response}

This cart met goals of a more ergonomic fit and increased the prone cart utility with the addition of convenience items such as a tray and cup holder. The split design with the ability to raise the torso and decline the leg pad gave the user support while driving the prone cart. Because users no longer needed to fully support and elevate their head, neck, and back while on the prone cart, the possible user profile was enlarged to allow per- sons with less strength and body function. The cup holder and tray were well received by users who enjoyed taking the prone cart to the VA canteen for snacks and drinks; the tray and cup holder were also helpful in art therapy. One user requested a larger tray to use with his laptop, which did not fit easily on the current tray design. While the support pad design was a better ergonomic fit to the user body, this iteration did not address goals of motorization and maneuverability. With the elevation of the torso, the user's arms were not able to easily push the cart manually. This new support pad design needed to be incorporated with a motorized system to be fully functional.

\section{Iteration 4: Commercial-Grade Prone Cart}

\section{Cart Specifications and Design Goals}

- Height: $78.75 \mathrm{~cm}$ tall, with $10 \mathrm{~cm}$ of additional adjustable height.

- Wheels: $30.5 \mathrm{~cm}$ rubber wheels, recessed.

- Mobility: E-fix motorized system.

- Frame: $70 \mathrm{~kg}, \mathrm{H}$-frame with split cart pad able to change angles.

- Design goal: Combination of ergonomic fit, maneuverability, motorization, and utility for functional independence.

\section{Design}

With each of the previous iterations addressing two of the four overarching design goals, interdisciplinary teams worked to create a combined prone cart that met all design goals. This team combined the strengths of the two previous prone cart iterations and incorporated some new components to create the commercial prone cart. The commercial-grade cart completed in 2013 incorporated the lightweight, E-fix motorized system on the $\mathrm{H}$ frame design with the divided split support pad allowing $40^{\circ}$ of elevation and $-30^{\circ}$ of declination (Figure 10). The commercial-grade cart used the E-fix system with $30.5 \mathrm{~cm}$ rubber wheels recessed below the $\mathrm{H}$-frame and tip supports with a $0^{\circ}$ turning radius. This prone cart was both motorized and maneuverable.

The support pad material was changed from an open-cell foam to a visco-foam pad that created a more comfortable pressure distribution for the user. The support pad was covered in a soft, durable material (Dartex, Dartex Coatings Ltd; Nottingham, United Kingdom). Cutouts for the arms and chin on the torso pad were used to increase the range of motion of the user's arms while 


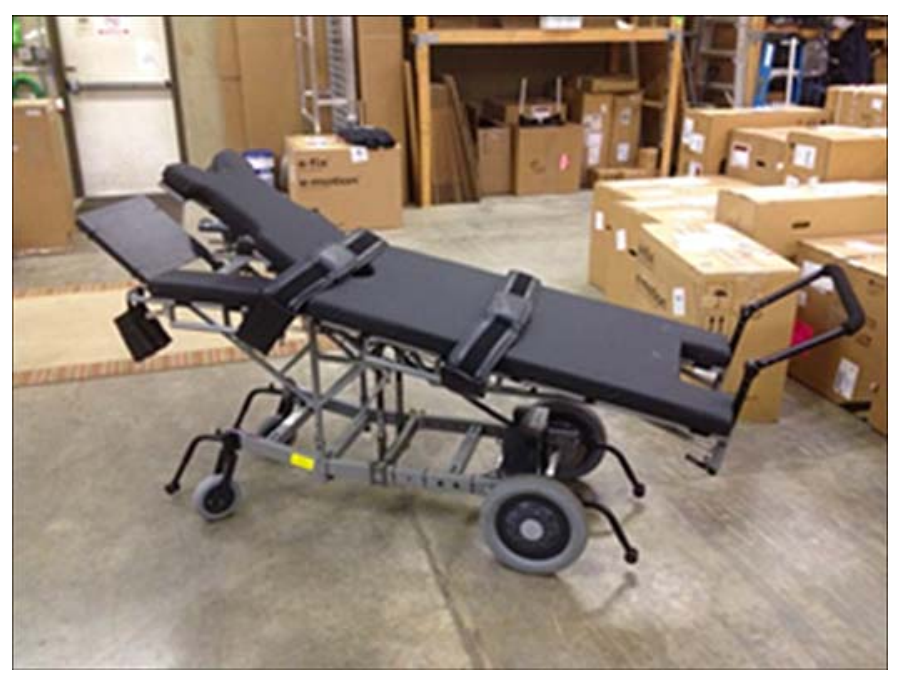

Figure 10.

Iteration 4: Commercial-grade prone cart.

on the cart and add better support to the torso. The divided split had a small area of horizontal pad to better fit the body when the torso was elevated and the legs were in the declined position. A cutout was added in the center of the cart to accommodate urinary catheters, which are worn by many users of the prone cart. The leg pad included a cutout for the feet with a strap along the bottom of the cart to secure the user's feet. Two Velcro straps were added to the middle sections of the cart to secure the user onto the cart and help prevent sliding. The cart was a better ergonomic fit for users.

The height of the cart was further increased to $78.75 \mathrm{~cm}$, adjustable by a further $10 \mathrm{~cm}$ to allow for bidirectional bed transfer. The cup holder and tray were also included in this design. The tray size was increased to accommodate more user functions, such as room for a laptop. These items increased the prone cart utility to support functional independence, because the ability to carry items is key to supporting the independence of users as opposed to being merely a luxury.

\section{User Response}

The new commercial-grade prone cart was used in the VA starting in January of 2014. Users and therapists at the VA noted the cart was easy to maneuver and more comfortable than previous prone carts. The height adjustment made transferring easier and safer for the user. The commercial prone cart design reduced the strain on the user's neck, back, and shoulders. Users at the VA were happy to have mobility independence during long stays for pressure ulcer treatment. The increased tray size had increased functionality for the user. Therapists at the VA provided feedback that they felt the new commercialgrade prone cart was ready to be recommended and used by a larger number of pressure ulcer patients, with the incorporation of a motorized component and better ergonomic design.

\section{Future Iteration}

The new commercial-grade prone cart offers a more ergonomic design, increased utility, more maneuverability, and a motorized component; design teams continue to revamp the prone cart. Potential changes for future carts include adjustments to the torso pad, cutout sizes, foot length, foam density, and rounding edges (Figure 11). A longer torso pad will allow for easier placement of the chin on the head rest and a better placement of the actual bend of the torso when the user is placed on the prone cart. The current torso pad is $58.5 \mathrm{~cm}$; the design team feels that $69 \mathrm{~cm}$ or even more will be a better fit for users of the prone cart. The openings for the catheter can be increased to allow for easier placement of the catheter while the prone cart is being used. The arm semicircles could also be increased from a radius of $30.5 \mathrm{~cm}$ to about $46 \mathrm{~cm}$ to allow for increased arm movement and comfort. The foot cutout in the rear of the prone cart can be extended more anteriorly to accommodate shorter users. By rounding the edges of the frame in the corners and

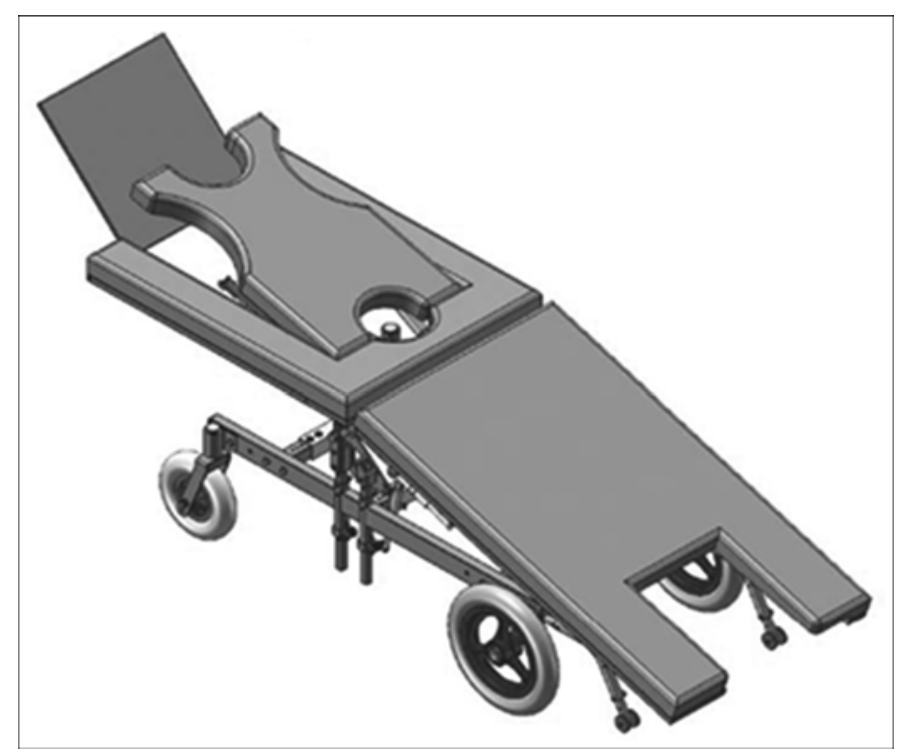

Figure 11.

Iteration 5: Potential future design iteration. 
extending the pad around the outline of the frame, it will be possible to prevent any injury on potentially sharp edges. The Dartex material, while soft and durable, tends to be slightly slippery according to user feedback and may be changed to a material with more grip to prevent sliding. Finally, the new prone cart foam may cause some degree of increased pressure on the knee of users while in use (Figure 12 and Figure 13). This may be particularly increased in persons with SCI who have hip contractures. There is a possibility that changes in foam density throughout the entire support pad could be used to redistribute the pressure from the knees to a larger area such at the thighs.

This project was approached from the standpoint of clinical quality improvement. Future design efforts may benefit from being approached as clinical research, with clinical trials being conducted. Clinical research trials of this sort may benefit from giving additional attention to the SCI level and severity of each user (i.e., American Spinal Injury Association score A through E), and the number of users enrolled in each iteration could be controlled for data analysis. Subject demographic factors could be analyzed in such a study. Clinical trials could use formalized questionnaires and patient evaluation protocols to guide design efforts; these results could then be compared and analyzed.

\section{CONCLUSIONS}

Interdisciplinary collaboration between medical professionals, engineers, and industry specialists combined with consistent user feedback led to an updated prone cart that incorporates motorization, maneuverability, and utility with comfort and ergonomic support. The consistent attention to user needs while creating a cart that can be easily and economically serviced has produced a motorized, ergonomic prone cart that can help improve user quality of life, increase compliance with pressure ulcer treatment recommendations, and offer more independence and mobility to users hospitalized at the VA. Through an iterative and collaborative process, the final commercial, motorized, prone cart successfully reached goals of motorization, maneuverability, ergonomic fit, and utility. While this cart addressed many of the problems inherent to the original prone cart design, the prone cart development is not finished. The continued collaboration and feedback from medical teams, engineers, industry specialists, and end users will help to keep the prone cart design current and relevant to user and therapy

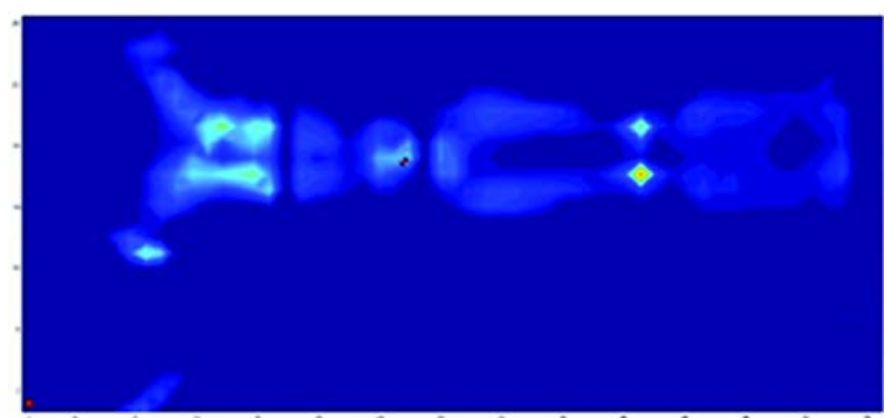

Figure 12.

Pressure mapping of iteration 4, highlighting potential areas of improvement.

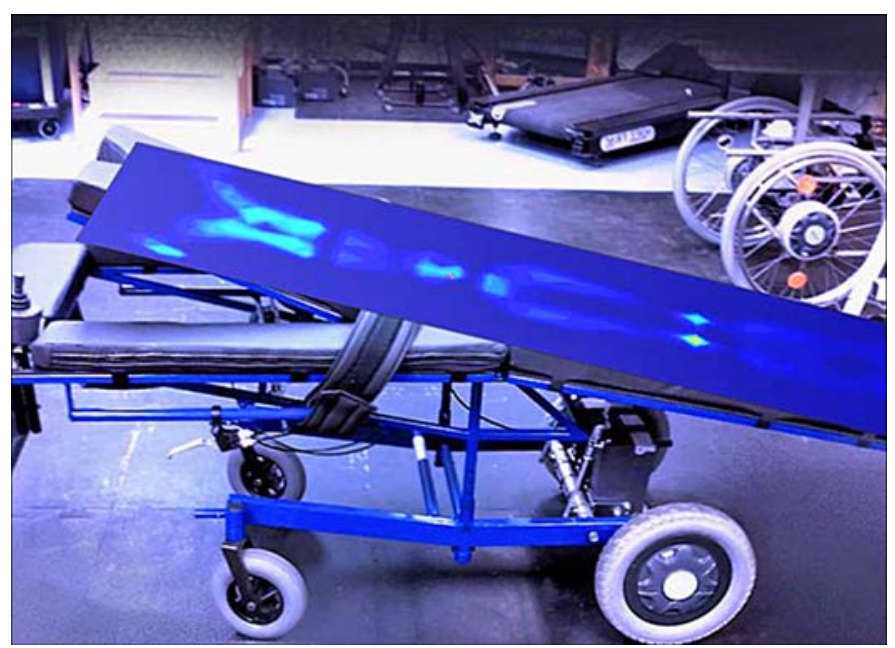

Figure 13.

Pressure mapping of iteration 4 overlaid onto a potential prone cart surface.

needs. Other facilities such as children's' hospitals have expressed interest in further prone cart designs. A pediatric prone cart for use after selective rhizotomy surgery is currently being designed, along with the adjustments to the current commercial adult prone cart design. Through collaboration and multiple design iterations, design teams created a cart that persons with SCI can use to maintain their independence during treatment of pressure ulcers.

\section{ACKNOWLEDGMENTS}

\section{Author Contributions:}

Drafting of manuscript: S. W. Brose, M. J. Kilbane, E. Harpster, S. J. Mitchell, C. Ho, K. J. Gustafson. 
Critical revision of manuscript for important intellectual content:

S. W. Brose, M. J. Kilbane, E. Harpster, S. J. Mitchell, C. Ho, K. J. Gustafson.

Study supervision: S. W. Brose, M. J. Kilbane, E. Harpster, S. J. Mitchell, C. Ho, K. J. Gustafson.

Administrative and technical support: S. W. Brose, M. J. Kilbane, E. Harpster, S. J. Mitchell, C. Ho, K. J. Gustafson.

Financial Disclosures: The authors have declared that no competing interests exist.

Funding/Support: This material was based on work supported by the VA Rehabilitation Research and Development Service (award 822, B6685).

Additional Contributions: Thanks to Nick Furlani and Josh Hodnichak for design and fabrication of design iteration 3.

\section{REFERENCES}

1. Bates-Jensen BM, Guihan M, Garber SL, Chin AS, Burns SP. Characteristics of recurrent pressure ulcers in veterans with spinal cord injury. J Spinal Cord Med. 2009;32(1):3442. [PMID:19264047]

2. Garber SL, Rintala DH. Pressure ulcers in veterans with spinal cord injury: A retrospective study. J Rehabil Res Dev. 2003;40(5):433-41. [PMID:15080228] http://dx.doi.org/10.1682/JRRD.2003.09.0433

3. McKinley WO, Jackson AB, Cardenas DD, DeVivo MJ. Long-term medical complications after traumatic spinal cord injury: A regional model systems analysis. Arch Phys Med Rehabil. 1999;80(11):1402-10. [PMID:10569434] http://dx.doi.org/10.1016/S0003-9993(99)90251-4

4. Backhaus M, Citak M, Tilkorn DJ, Meindl R, Schildhauer TA, Fehmer T. Pressure sores significantly increase the risk of developing a Fournier's gangrene in patients with spinal cord injury. Spinal Cord. 2011;49(11):1143-46.

[PMID:21788955]

http://dx.doi.org/10.1038/sc.2011.75

5. Henzel MK, Bogie KM, Guihan M, Ho CH. Pressure ulcer management and research priorities for patients with spinal cord injury: Consensus opinion from SCI QUERI Expert Panel on Pressure Ulcer Research Implementation. J Rehabil Res Dev. 2011;48(3):xi-xxxii. [PMID:21480093] http://dx.doi.org/10.1682/JRRD.2011.01.0011

6. Citak M, Backhaus M, Tilkorn DJ, O’Loughlin PF, Meindl R, Muhr G, Fehmer T. Necrotizing fasciitis in patients with spinal cord injury: An analysis of 25 patients. Spine. 2011; 36(18):E1225-29. [PMID:21325991]

http://dx.doi.org/10.1097/BRS.0b013e3182059950

7. Chan BC, Nanwa N, Mittmann N, Bryant D, Coyte PC, Houghton PE. The average cost of pressure ulcer management in a community dwelling spinal cord injury population. Int Wound J. 2013;10(4):431-40. [PMID:22715990] http://dx.doi.org/10.1111/j.1742-481X.2012.01002.x

8. Ho CH, Bogie K. The prevention and treatment of pressure ulcers. Phys Med Rehabil Clin N Am. 2007;18(2):235-53. [PMID:17543771] http://dx.doi.org/10.1016/j.pmr.2007.02.004

9. Nelson A, Malassigné P, Cors M, Amerson TL, Bonifay R, Schnurr E. Patient evaluation of prone carts used in spinal cord injury. SCI Nurs. 1996;13(2):39-44. [PMID:8900708]

10. Harrow JJ, Malassigné $P$, Nelson AL, Jensen RP, Amato M, Palacios PL. Design and evaluation of a stand-up motorized prone cart. J Spinal Cord Med. 2007;30(1):50-61. [PMID:17385270]

11. Malassigné P, Nelson AL, Cors MW, Jensen RP, Amato M, Schnurr ES, Amerson TL. Iterative design and evaluation of new prone carts for individuals with SCDs: A technical note. J Rehabil Res Dev. 2002;39(1):127-39. [PMID:11930905]

12. Brose SW, Wali E. Multidisciplinary approach to converting power chair into motorized prone cart. J Rehabil Res Dev. 2014;51(9):1377-82. [PMID:25786192]

http://dx.doi.org/10.1682/JRRD.2014.01.0027

Submitted for publication November 7, 2014. Accepted in revised form September 11, 2015.

This article and any supplementary material should be cited as follows:

Brose SW, Kilbane MJ, Harpster E, Mitchell SJ, Ho C, Gustafson KJ. Interdisciplinary development of an ergonomic prone mobility cart. J Rehabil Res Dev. 2016; 53(4):433-42.

http://dx.doi.org/10.1682/JRRD.2014.11.0279

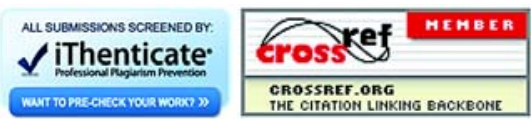

